\title{
Disrupted Activity in the Hippocampal-Accumbens Circuit of Type III Neuregulin I Mutant Mice
}

\author{
Malcolm W Nason Jr', Avishek Adhikari' ${ }^{2}$, Marjan Bozinoski', Joshua A Gordon ${ }^{*, 3,4}$ and Lorna W Role*1,3 \\ 'Department of Neurobiology and Behavior and Center for Nervous System Disorders, SUNY Stony Brook University, Stony Brook, NY USA; \\ ${ }^{2}$ Department of Biology, Columbia University, New York, NY, USA; ${ }^{3}$ Department of Psychiatry, Columbia University, New York, NY, USA; \\ ${ }^{4}$ Department of Integrative Neuroscience, New York State Psychiatric Institute, New York, NY, USA
}

\begin{abstract}
Neuregulin I ( $\mathrm{Nrg}$ /), a schizophrenia susceptibility gene, is involved in fundamental aspects of neurodevelopment. Mice lacking any one of the several isoforms of $\mathrm{Nrg}$ I have a variety of schizophrenia-related phenotypes, including deficits in working memory and sensorimotor gating, loss of spines in pyramidal neurons in the ventral subiculum, loss of dendrites in cortical pyramidal cells, loss of parvalbuminpositive interneurons in the prefrontal cortex, and altered plasticity in corticolimbic synapses. Mice heterozygous for a disruption in exon 7 of the $\mathrm{Nrg}$ I gene lack Type III (cysteine-rich-domain-containing) isoforms and have sensorimotor gating deficits that may involve changes in the activity of a circuit involving projections from the ventral hippocampus (VHPC) to medium spiny neurons in the nucleus accumbens (nACC). To explore the neural basis of these deficits, we examined electrophysiological activity in the nACC and vHPC of these mice. Under urethane anesthesia, bursts of spontaneous activity propagated from the vHPC to the nACC in both wild-type and mutant mice. However, these bursts were weaker in mutant nACC, with reduced local field potential amplitude and spiking activity. Single units in mutant nACC fired less frequently within the bursts, and more frequently outside of the bursts. Moreover, within-burst nACC spiking was less modulated by vHPC activity, as determined by phase-locking to the low-frequency oscillatory components of the bursts. These data suggest that the efficacy of VHPC input to the nACC is reduced in the Type III Nrg I heterozygotes, supporting a role for $\mathrm{Nrg}$ I in the functional profile of hippocampal-accumbens synapses.

Neuropsychopharmacology (20II) 36, 488-496; doi:I0.1038/npp.2010.180; published online 6 October 2010
\end{abstract}

Keywords: hippocampus; nucleus accumbens; neuregulin; functional connectivity; schizophrenia; limbic system

\section{INTRODUCTION}

The nucleus accumbens (nACC) is a critical mediator of limbic motor computations. The nACC receives inputs from the ventral hippocampus (vHPC), prefrontal cortex, ventral tegmental area, and amygdala, and is thus in a privileged position to have a role in context-informed volitional behaviors (Groenewegen et al, 1987; Wright and Groenewegen, 1995; Mulder et al, 1998; Groenewegen et al, 1999; O'Donnell et al, 1999; Grace, 2000; French and Totterdell, 2002, 2003; Groenewegen and Trimble, 2007; Sesack and Grace, 2010). The interaction of these numerous afferents within the nACC has been implicated in circuits controlling sensory-motor gating and working memory, behaviors

\footnotetext{
*Correspondence: Dr JA Gordon, Department of Psychiatry, Columbia University, I05I Riverside Drive Unit 87, Kolb Annex L174, New York, NY 10032, USA, Tel: + I 212543 6768, Fax: + | 212543 I I74, E-mail: jg343@columbia.edu or Dr LW Role, Department of Neurobiology and Behavior, SUNY Stony Brook, Stony Brook, NY | 1794-5230, USA, Tel: + | 631632 4100, Fax: + | 631 632-6661, E-mail: Lorna.Role@stonybrook.edu

Received 29 April 2010; revised 3 September 2010; accepted 4 September 2010
}

that are typically disrupted in patients with schizophrenia (Braff and Geyer, 1990; O’Donnell and Grace, 1998; Freedman et al, 2003; Barch, 2005; Simpson et al, 2010).

Although the causal pathophysiology of schizophrenia is not well understood, it is clear that genetic susceptibility factors have an important role. One such factor is the gene for neuregulin 1 (Nrg1), which has been linked to schizophrenia in multiple, independent linkage studies (Stefansson et al, 2002; Harrison and Weinberger, 2005; Harrison and Law, 2006; Li et al, 2006; Munafo et al, 2006; Nicodemus et al, 2009). A complex, variably spliced molecule, Nrg1 has been implicated in the regulation of diverse processes, including neuronal survival, neuronal migration, axonal pathfinding, and peripheral myelination (Michailov et al, 2004; Taveggia et al, 2005; Lopez-Bendito et al, 2006; Bjarnadottir et al, 2007; Krivosheya et al, 2008; Chen et al, 2010). Of the various classes of Nrg1 isoforms, the Type III Nrg1 variety is of particular interest. Type III Nrg1 affects synaptic function by acting as both a ligand of and a receptor for ErbB receptor tyrosine kinases, inducing bidirectional signaling in a juxtacrine manner (Bao et al, 2003; Hancock et al, 2008). Mice with specific disruptions in exon 7 of the Nrg1 gene $\left(\mathrm{Nrg}^{\text {tmlLwr }}+/\right.$ - or Type III Nrg1 $\left.{ }^{+/-}\right)$ 
have decreased expression of Type III Nrg1 isoforms, and are deficient in sensory-motor gating and spatial working memory behaviors (Wolpowitz et al, 2000; Chen et al, 2008), similarly to patients with schizophrenia (Thaker, 2000). Analyses of corticolimbic (eg, cortex to basolateral amygdala and vHPC to nACC) circuits in vitro reveal altered synaptic plasticity in Type III $\mathrm{Nrg}^{+/-}$compared with control (Zhong et al, 2008). Given these in vitro findings and the behavioral impairments in sensorimotor gating and memory in Type III $\mathrm{Nrg}^{+/-}$mice (Chen et al, 2008), we tested the hypothesis that disruption of Type III Nrg1 signaling would alter the profile of vHPC-nACC circuits in vivo. Here we examine the effect of altered Type III Nrg1 expression in vHPC-nACC circuits in vivo by recording simultaneously from the nACC and vHPC in urethane-anesthetized wild-type (WT) and Type III $\mathrm{Nrg} 1^{+/-}$mice. Consistent with previous findings (Goto and O'Donnell, 2001a,b) we describe bursts of spontaneous neural activity in the nACC, which follow similar bursts in the vHPC in mice of both genotypes. Characterizing nACC unit and local field potential (LFP) data in and around these bursts, we show that decreased expression of Type III Nrg1 disrupts the temporal organization of nACC activity, consistent with diminished efficacy of inputs from the vHPC to the nACC. These results support the notion that changes in functional connectivity in the vHPC-nACC circuit may underlie the deficits in sensory-motor gating and spatial working memory behaviors displayed by Type III $\mathrm{Nrg} 1^{+/-}$mice.

\section{MATERIALS AND METHODS}

\section{Animals}

Type III Nrg1 heterozygous mutant mice $\left(\mathrm{Nrg} 1^{\text {tm1Lwr }}+/-\right.$, referred to here as Type III $\mathrm{Nrg}^{+/-}$) were back crossed to $>99.9 \%$ C57Bl6 background. Mutant and WT littermates were generated by crossing Type III $\mathrm{Nrg}^{+1-} \times$ Type III $\mathrm{Nrg} 1^{++-}$or Type III Nrg1 ${ }^{+/-} \times \mathrm{C} 57 / \mathrm{Bl6}$ WT as described (Wolpowitz et al, 2000). Animals were maintained on a 12-h light/dark cycle and provided with food and water ad libitum. Animal testing was conducted in accordance with the Principles of Laboratory Animal Care and the guidelines of the National Institutes of Health and was approved by the State University of New York at Stony Brook, Columbia University and New York State Psychiatric Institute Institutional Animal Care and Use Committees.

\section{Surgical Procedure}

WT and Type III $\mathrm{Nrg}^{+/-}$mice $(30-40 \mathrm{~g} ; n=6 \mathrm{WT}, 13$ mutants) were anesthetized with $200 \mathrm{mg} / \mathrm{kg}$ urethane (Sigma, St Louis, MO), and a tracheostomy was performed for insertion of custom-made glass tubes (1.2 mm OD, WPI, Sarasota, FL) to maintain a patent airway. Animals were positioned in a stereotaxic device (David Kopf Instruments, Tujunga, CA) and the skull leveled. Burr holes were drilled (Foredom, Bethel, CT) over the nACC ( + $1 \mathrm{~A} / \mathrm{P}, 1-1.5 \mathrm{M} / \mathrm{L})$ and the vHPC $(-3.9 \mathrm{~A} / \mathrm{P}, 2.2-2.5 \mathrm{M} / \mathrm{L})$. Core temperature was maintained at $37^{\circ} \mathrm{C}$ by a feedback-controlled device (Warner Instruments, Hamden, CT) and 100\% humidified oxygen was blown past the tracheotomy tube. Animals were allowed to equilibrate for $\geqslant 1 \mathrm{~h}$ before cell search began. nACC was searched by differential recording through two tungsten recording electrodes (5 M, 0.01 inch; A-M Systems, Carlsborg, WA) glued together with cyanoacrylate. The electrode tips were configured in an offset manner in both the vertical and horizontal directions. One tip was $\sim 400 \mu \mathrm{m}$ lower, and $\sim 400 \mu \mathrm{m}$ lateral to the other, with the lower/medial tip placed in the nACC and the upper/lateral tip used for a reference. A similar offset recording/reference pair was positioned in the vHPC, except that the recording electrode was placed laterally and ventrally relative to the reference electrode. The use of different local references in each brain region to record the field potentials in the nACC and vHPC reduces the likelihood that the results can be accounted for by volume conduction or contamination of common noise from the reference electrode.

\section{Cell Recording Protocol}

Electrodes were lowered into the target regions until spontaneously active units were identified. Successfully isolated units were recorded for a minimum of $15 \mathrm{~min}$. For LFP recordings, data were filtered $(0.5-200 \mathrm{~Hz})$, and digitized at $2 \mathrm{kHz}$ using Spike2 acquisition software (CED, Cambridge, UK). Cell data were acquired at $40 \mathrm{kHz}$ using Spike2; spikes were threshold-detected with a minimum signal-to-noise ratio of $4: 1$. Waveforms were digitized (36 points per waveform). Individual waveforms were discriminated off-line using template-matching software (Spike2), which was then confirmed by Cluster Analysis (Spike2). LFPs were recorded from the same electrodes, Bessel filtered $(0-100 \mathrm{~Hz}$ bandpass), and acquired at $2 \mathrm{kHz}$. After recording, anodal current was passed $(20 \mu \mathrm{A}$ for $1 \mathrm{~min})$, marking the site of the recording. Only one recording site was utilized per animal. Animals were overdosed with urethane, and perfused transcardially with cold PBS followed by $10 \%$ formalin. After $10 \mathrm{~min}$ of perfusion fixation, the brain was removed and stored in $10 \%$ formalin overnight. Brains were then immersed in $30 \%$ sucrose in $0.1 \mathrm{M}$ PBS until equilibration. Samples were sectioned $(50 \mu \mathrm{m})$ in the coronal plane, and marked recording sites were visualized and plotted on standard sections. All cells included in this study were from the shell region of the nACC (Figure 1).

\section{Data Analysis}

Cellular burst analysis was completed with a built-in Spike2 'Burst' algorithm. Parameters were set so that bursts comprised a minimum of three spikes, were initiated when instantaneous firing rate reached $10 \mathrm{~Hz}$, and were terminated when firing rate decreased below $3 \mathrm{~Hz}$. Bursts had to be separated by a minimum of $50 \mathrm{~ms}$ of silence. These parameters were selected by qualitative analysis of a representative selection of data and were determined to be non-restrictive, including most high-frequency events (Cooper and White, 2000) in the Type III Nrg1 ${ }^{+/-}$mice.

nACC LFP recordings highlighted low-frequency, highamplitude oscillations concurrent with spike bursts (Figure 2). These were termed 'LFP bursts'. LFP bursts were identified by a Spike2 thresholding algorithm that detected changes in the LFP that were biphasic, at least two 

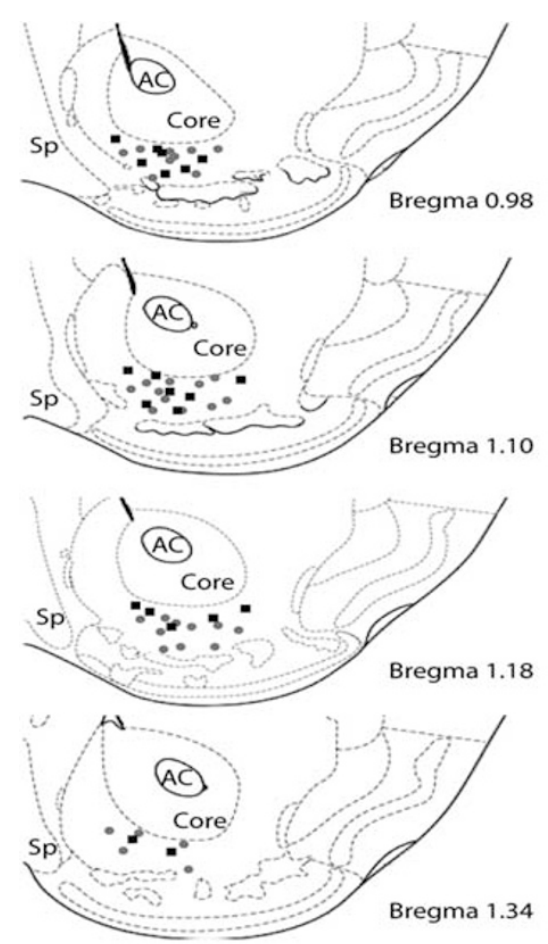

- Wild Type
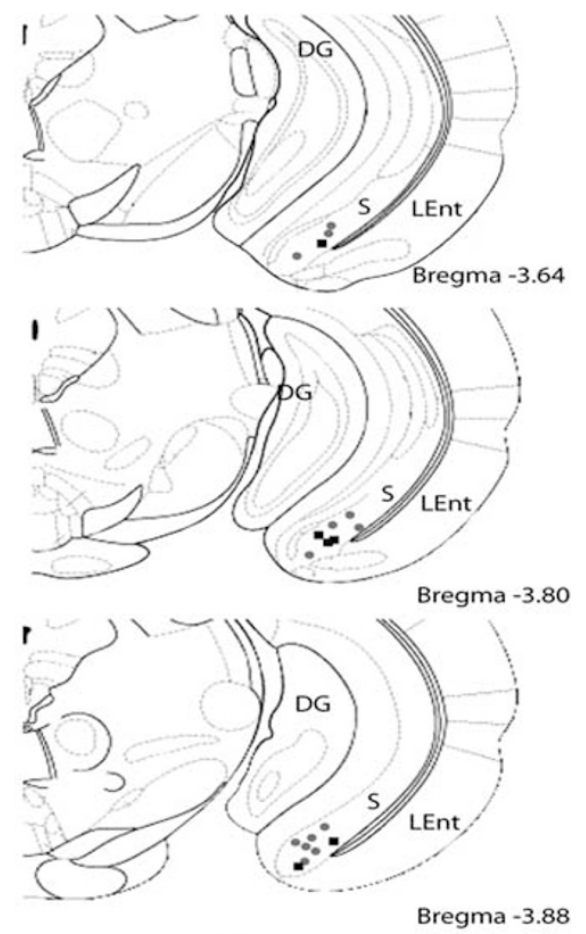

Figure I Recording locations for all experiments. Recording locations from nACC (left panels) and VHPC (right panels) from WT (black squares) and Type III NrgI ${ }^{+/-}$(red circles) mice superimposed onto standard atlas schematics. AP distance from the bregma in millimeter is indicated at lower right of each panel. Positive numbers are anterior to the bregma. AC, nACC; DG, dentate gyrus; LEnt, lateral entorhinal cortex; S, ventral subiculum; Sp, piriform cortex.

times the standard deviation of absolute LFP power, and began with a negative-going voltage component. After finding these LFP waves, the algorithm set the start time of the LFP burst as the zero-crossing point before the negative-going portion of the biphasic LFP waveform. Algorithm precision was cross-checked by direct inspection of all LFP burst start points by the investigator. Start points judged incorrect were reset by filtering the LFP signal from 0.5 to $4 \mathrm{~Hz}$, re-running the thresholding algorithm and marking the start points as the time the filtered LFP crossed $0 \mathrm{mV}$. Peak and trough amplitudes of each LFP burst were detected and averaged to compare LFP burst sizes across genotypes. Peri-burst time histograms were constructed by using the start times of nACC LFP bursts to compile nACC cellular spike time histograms. LFP peri-burst power ratios compared the oscillatory power immediately previous to the start of the LFP burst with the power during the LFP burst. The 1-s time windows were constructed based upon the start times (see above) of each LFP burst yielding a 1-s 'preburst' period and a 1-s 'burst' period. Raw LFP waves were filtered for $\delta(0.1-4 \mathrm{~Hz}), \theta(4-12 \mathrm{~Hz})$, and $\gamma(30-90 \mathrm{~Hz})$. Power in the $\theta$ and $\gamma$ bandwidths was computed by a multitaper method (MATLAB (Mathworks, Natick, MA); seven non-overlapping windows) and $\delta$ power was quantified as the size of the area under the filtered LFP for each $1 \mathrm{~s}$ period of time (pre and burst) for every LFP burst. Each peri-burst measurement was computed as a ratio (burst/ pre-burst) to normalize for LFP amplitude differences. Results are stated as the average ratio by animal.
Spike phase-locking and LFP lag analysis were conducted using custom scripts written in MATLAB (Mathworks). The strength of single-unit phase-locking to $\theta$ oscillations was assessed using Rayleigh's $Z$-statistic of circular uniformity. To determine whether spikes were phase-locked to $\theta$, instantaneous, $\theta$ phases of LFPs were determined through the Hilbert transform on the $\theta$-filtered vHPC LFP, and a phase was assigned to each spike based on the time of the spike's occurrence. A phase of 0 refers to the trough of the $\theta$ cycle as recorded, although the variability in placement of the electrodes precludes assigning absolute phase relative to known landmarks. Rayleigh's $Z$-statistic values were obtained by the analysis of phase-locking of 100 randomly chosen spikes from a minimum of $900 \mathrm{~s}$ of experimental time. To ensure a random sample of spikes, this process was repeated 1000 times per experiment. The final $Z$-value reported is the average $Z$-statistic of these 1000 phase-locking iterations.

To determine whether vHPC leads nACC activity or vice versa, two different methods were used. First, to determine the temporal relationship between single-unit activity and vHPC $\theta$ oscillations, phase-locking was calculated for 80 different temporal offsets (in $2.5 \mathrm{~ms}$ increments) for each unit recording. Units with significant Bonferroni-corrected phase-locking in at least 1 of the 80 shifts were used for the analysis in Figure 6c. No mutant units were significantly phase-locked using this stringent criterion. Negative lags indicate vHPC leading the nACC. Second, to determine the temporal relationship between the low-frequency 
a
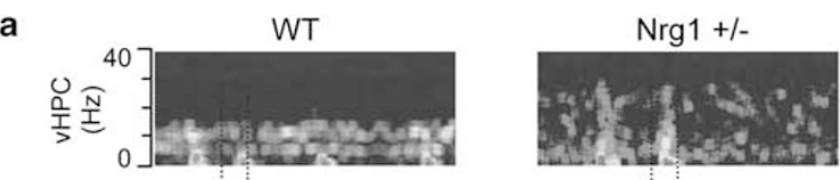

b

C

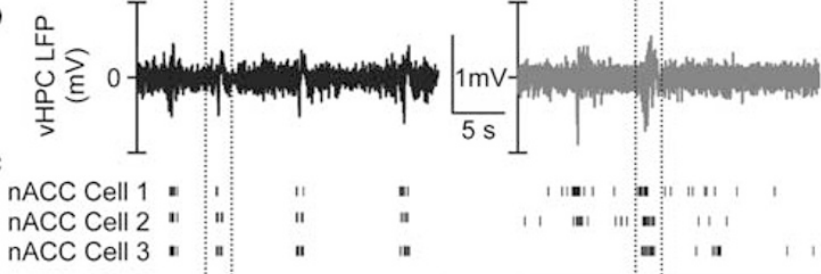

d

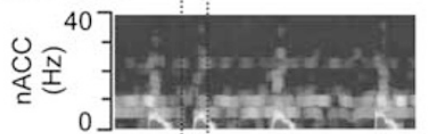

e

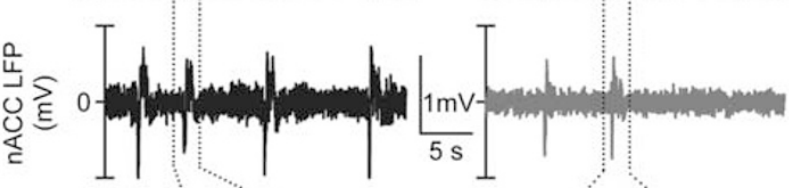

f

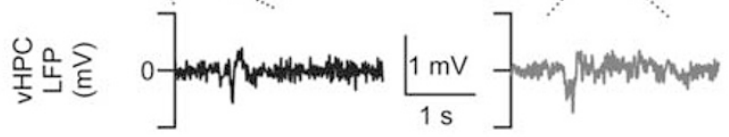

g nACC Cell 1
nACC Cell 2
nACC Cell 3

h

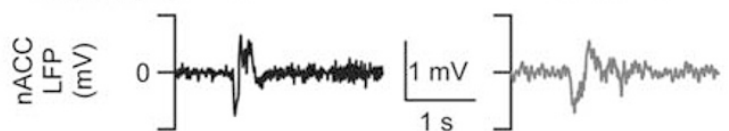

Figure 2 nACC activity is disorganized in Type III Nrg| ${ }^{+/-}$mice. Spectrograms and LFP recordings from the VHPC ( $a$ and $b$ ) and nACC (d, e) reveal synchronous bursts of low-frequency power in both brain regions. (c) Raster plots of nACC unit activity. Note the staccato, bursting discharge pattern of nACC cells in the WT (left panels) and the less precise organization of spikes from nACC cells in the Type III Nrg| ${ }^{+/-}$mouse (right panels). ( $f-h)$ These simultaneous events are seen in expanded views showing the structure of the cellular discharge $(\mathrm{g})$ and the LFP bursts recorded in the VHPC (f) and $\mathrm{nACC}(\mathrm{h})$.

components of the LFP, we filtered each LFP from 0.1 to $50 \mathrm{~Hz}$ and obtained the instantaneous amplitude using the Hibert transform. We then cross-correlated the instantaneous amplitudes of vHPC and nACC and measured the lag to the peak of the cross-correlation (Adhikari et al, 2010). Negative lags indicate that vHPC leads nACC.

\section{RESULTS}

\section{Disorganized Patterns of Neural Activity in Type III Nrg1 ${ }^{+/-}$nACC}

Recordings of neural activity were obtained from the nACC and vHPC of $6 \mathrm{WT}$ and 13 Type III $\mathrm{Nrg} 1^{+/-}$mice under urethane anesthesia. High-impedance electrodes and standard spike sorting techniques were used to record spontaneous activity from $21 \mathrm{WT}$ and 35 mutant nACC neurons. Simultaneous LFP recordings were obtained from the CA1 pyramidal layer of the vHPC. Mean nACC neuron firing rates $(19.8 \pm 2.2 \mathrm{~Hz}$ for WT $v s 17.4 \pm 1.4$ for Type III $\left.\mathrm{Nrg} 1^{+/-}, p=0.5\right)$ did not differ by genotype.

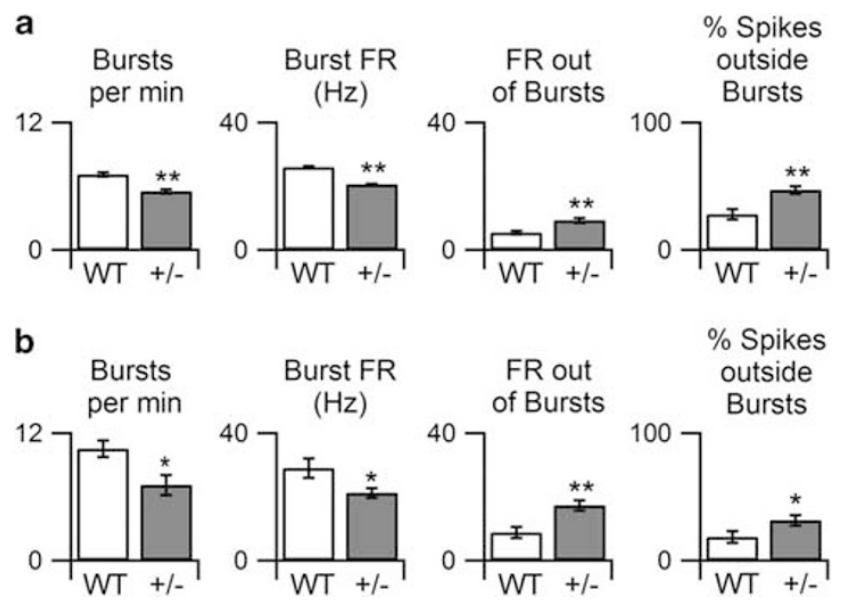

Figure $3 \mathrm{nACC}$ neuronal firing patterns are altered in Type III Nrg | ${ }^{+-}$ mice. ( $a$ and $b$ ) Left to right: Mean \pm SEM burst rate, intraburst spike rate, extraburst spike rate, and \% of spikes occurring outside of bursts in WT (white bars) and Type III Nrgl ${ }^{+1-}$ (gray bars) mice, for cell bursts (a), defined by short interspike intervals in the spike record, and LFP bursts (b), defined by the occurrence of increased low-frequency power in the LFPs. * $p<0.0$ I, ** $p<0.00$ I.

Recordings of nACC and vHPC activity in WT animals suggested a high degree of temporal coordination across areas. The discharge pattern of nACC cells in WT animals was characterized by discrete staccato bursts of spikes interspersed with long, interburst pauses (Figure $2 \mathrm{c}$ and g; left-side panels). nACC spike bursts were accompanied by large amplitude, low-frequency LFP events (Figure 2d, e and h). Similar large-amplitude, low-frequency LFP events were recorded in the vHPC (Figure $2 \mathrm{a}, \mathrm{b}$ and $\mathrm{f}$ ), which coincided with LFP waves in the nACC. The LFP events in both locations consisted of a stereotyped bi- or triphasic waveform with high $\delta-(1-4 \mathrm{~Hz})$ and $\theta-(4-12 \mathrm{~Hz})$ range power (Figure $2 \mathrm{a}$ and $\mathrm{d}$ ).

Although nACC and vHPC activity in Type III $\mathrm{Nrg}^{+/-}$ mice displayed a pattern of bursts of cellular activity accompanied by large, low-frequency LFP events that was similar to that of WT mice, the frequency and coordination of this activity was changed considerably in the Type III $\mathrm{Nrg} 1^{+/-}$recordings compared with WT (Figure $2 \mathrm{a}-\mathrm{h}$; rightside panels). Activity bursts in the Type III Nrg1 ${ }^{+/-}$mice appeared to occur less often, and spiking activity within the bursts appeared to be less tightly correlated with the LFP events in either area. To quantify these differences, two independent methods were used to identify activity bursts. First, spike bursts of nACC single units were categorized by an interspike interval algorithm classifying the start of a burst as the point where unit firing frequency rose above $10 \mathrm{~Hz}$, and the end of the burst as the point where firing frequency fell below $3 \mathrm{~Hz}$. Second, nACC LFP bursts were identified by an amplitude-thresholding algorithm (see Materials and Methods). Results yielded by both methods were similar. Bursts of activity in the nACC, whether distinguished by cell discharge frequency or LFP events, occurred less frequently in Type III $\mathrm{Nrg} 1^{+/-}$ mutants than in WT animals (Figure $3 a$ and b). During these bursts, nACC single units from Type III Nrg1 ${ }^{+/-}$mice discharged at a lower rate and produced fewer spikes per burst $(6.9 \pm 0.1$ vs $5.8 \pm 0.05 ; p<0.001)$ relative to WT mice. In contrast, outside of these bursts, firing rates of nACC 
a

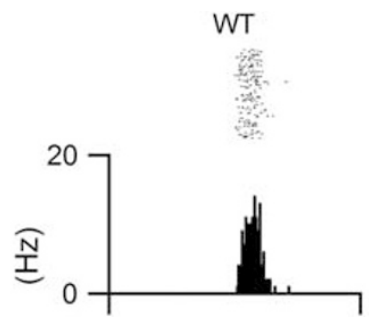

C

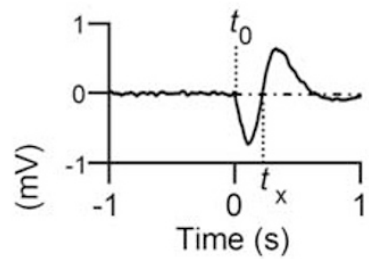

$\mathrm{Nrg} 1+/-$
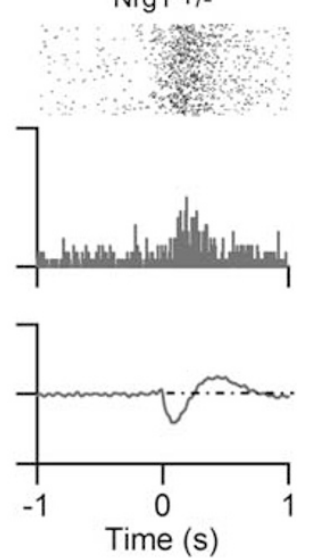

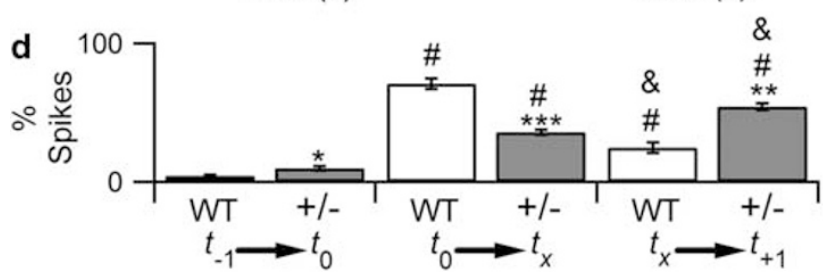

Figure 4 nACC neuronal burst firing patterns are altered in Type III $\mathrm{Nrgl}{ }^{+/-}$mice. (a-c) Peri-burst firing patterns from representative WT (left panels) and Type III Nrgl ${ }^{+/}$(right panels) units. (a) Raster plots of unit firing during $3 \mathrm{I}$ and 33 bursts for WT and Type III Nrg| ${ }^{+-}$examples, respectively. (b) Peri-burst time histograms averaged over these bursts. (c) Average burst signal in the LFP, triggered at the initiation of the burst in the LFP and averaged over these bursts. $t_{0}$, burst start; $t_{x}$, first zero crossing after $t_{x}$. (d) Within-burst firing patterns in WT and Type III Nrg| ${ }^{+/-}$mice averaged over all units. Percentage of peri-burst spikes that occur within the I s preceding the LFP burst ( $t_{-1}$ to $t_{0}$ ); between the beginning of the LFP burst and the first zero-crossing ( $t_{0}$ to $t_{x}$ ), and from the first zero crossing to Is after the LFP burst initiation $\left(t_{x}\right.$ to $\left.t_{+1}\right)$. * $p<0.05$, *** $p<0.0$, ***** $p<0.00$ I; WT vs + I- Type III Nrgl I I- "\# $p<0.0$ I; vs 't $t_{-1}$ to to' bin; and ${ }^{\&} p<0.0$ I; 't $t_{0}$ to $t_{x}$ ' bin vs 't $t_{x}$ to $t_{+}$' ' bin.

neurons were significantly higher in Type III $\mathrm{Nrg} 1^{+/-}$mice than in WT mice (Figure $3 a$ and $b$ ). Relatively few spikes from WT cells were found to occur outside of bursts, whereas almost half of all discharge events in Type III $\mathrm{Nrg} 1^{+/-}$units occur outside of bursts (Figure $3 \mathrm{a}$ and $\mathrm{b}$ ). To ensure the reliability of the unit analysis, cells were pooled by animal and the above measures re-tested on a per animal basis. All results remained significantly different. Hence, the temporal organization of nACC spike bursting is disrupted in Type III $\mathrm{Nrg} 1^{+/-}$animals as compared with WT mice.

To further explore the organization of nACC neuron firing, we examined the dynamics of unit discharge throughout the course of LFP events. Rasters and periburst time histograms time-locked to the start of LFP events (Figure $4 \mathrm{a}$ and $\mathrm{b}$ ) revealed a temporally precise onset and rapid offset of firing in WT nACC units. nACC neurons from Type III Nrg1 ${ }^{+/-}$mice, however, showed altered cellLFP synchronization. Considerable firing outside of bursts occurred in the mutants, and the neurons took longer to reach peak firing rate and maintained elevated firing rates throughout and beyond the LFP event. To quantify this effect, we compared the relative firing rates of nACC neurons in WT $v s$ Type III Nrg1 ${ }^{+/-}$mice across the periburst period. For each individual LFP event, unit discharge was compared between three distinct periods describing the

shape of the LFP event: the $1 \mathrm{~s}$ before the start of the LFP event $\left(t_{-1}\right.$ to $t_{0}$, period 1$)$, from the start of the event to the subsequent zero $\mathrm{mV}$ crossing ( $\mathrm{t}_{0}$ to $\mathrm{t}_{\mathrm{x}}$, period 2 ), and from the zero crossing to $1 \mathrm{~s}$ after the start $\left(t_{x}\right.$ to $t_{+1}$, period 3$)$. Spikes in each period were counted and normalized to total spikes over the entire $2 \mathrm{~s}$ event period (Figure $4 \mathrm{c}$ and $\mathrm{d}$ ). nACC units from Type III Nrg1 ${ }^{+}$mice had higher activity before the start of the LFP events, yet had fewer spikes before the zero crossing, and more spikes after the zero crossing than neurons from WT animals. This template of activity is consistent with (1) increased extraburst firing, (2) slower onset, and (3) slower offset of spiking activity in Nrg1 heterozygous mice compared with WT. Therefore, the composition of burst discharge patterns of nACC neurons is disorganized in Type III Nrg1 $1^{+/-}$mice.

In addition to the evidence from nACC single-unit firing patterns, comparison of the simultaneously recorded LFP activity in WT and Type III Nrg1 $1^{+/-}$mice also revealed significant differences in the mutants. As noted above, spiking activity was accompanied by stereotyped large amplitude LFP bursts in WT and Type III Nrg1 $1^{+/-}$mice. However, nACC LFP bursts in Type III Nrg1 ${ }^{+/-}$mice were considerably smaller than those in WT mice. The peak and the trough amplitude of the waves recorded in nACC of Type III Nrg1 ${ }^{+7-}$ mice were significantly reduced compared with those from WT mice (Figure 5a and b). In contrast, there was a nonsignificant trend toward an increase in the amplitude of LFP bursts recorded from the vHPC between genotypes (Figure $5 \mathrm{a}$ and $\mathrm{b}$ ), indicating that the mutant animals appear to have decreased nACC activity that is not due to decreases in the strength of vHPC bursts. Similar results were obtained in the frequency domain, comparing LFP power during and immediately preceding the bursts (Supplementary Figure S1).

\section{Decreased Functional Connectivity between the vHPC to the nACC in Type III Nrg1 ${ }^{+/-}$Mice}

The nACC receives direct monosynaptic input from the vHPC (Pennartz and Kitai, 1991; Mulder et al, 1998). We wondered whether the decreased nACC LFP burst amplitude and disorganization of spiking activity in the Type III $\mathrm{Nrgl}^{+/-}$mice might be the result of a deficit in the functional connectivity of vHPC inputs to the nACC. To explore this possibility, we examined the relationship between neural activity in the nACC and vHPC. We first explored the timing of the LFP bursts in the two regions. LFPs from the two regions were filtered for low frequencies $(1-50 \mathrm{~Hz})$ and instantaneous amplitude was calculated using the Hilbert transform. In both Type III Nrg1 ${ }^{+/-}$ and WT mice, cross-correlations of low-frequency amplitude envelopes (Adhikari et al, 2010) from nACC and vHPC showed a peak at negative lags, indicating that changes in amplitude in the vHPC precede those in the nACC (Figure 5c). Peak lags in the two genotypes were not different by $t$-test ( $p=0.4$ ) (Figure $5 \mathrm{~d}$ ). This result suggests that under these recording conditions, the vHPC leads the nACC.

To further study the possible directionality of the circuit, we examined the relationship between nACC unit discharge and the $\theta$-frequency components of the vHPC lowfrequency bursts. The $\theta$-frequency oscillations have been 

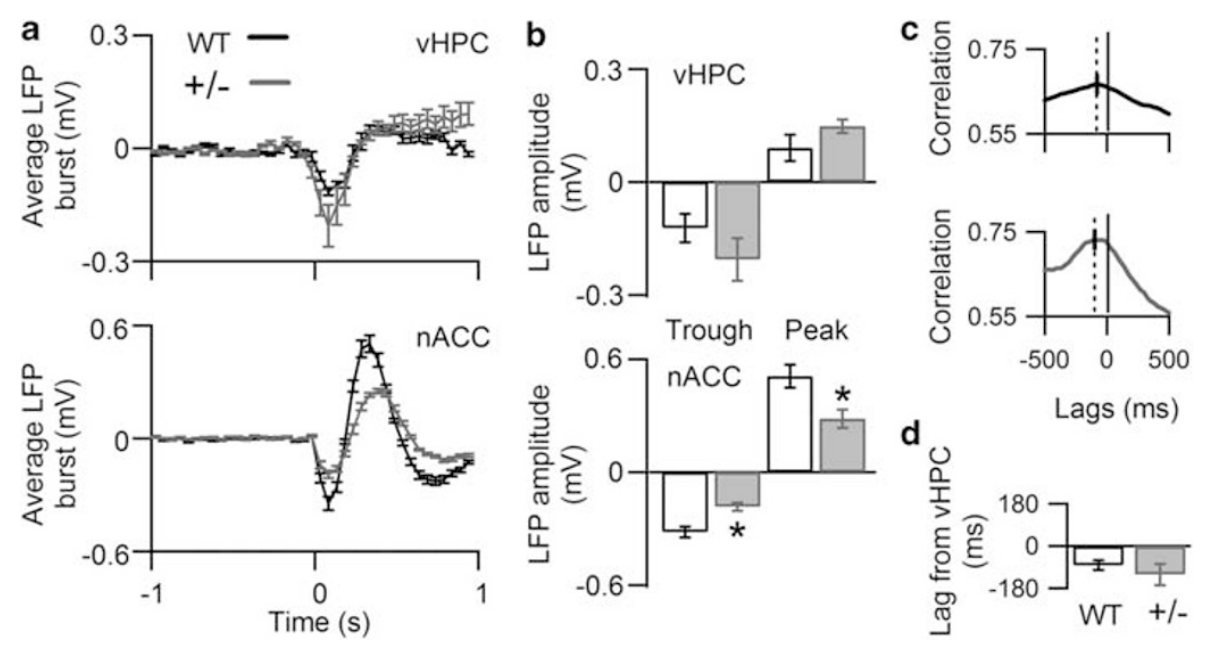

Figure 5 nACC LFP bursts reflect vHPC input and are weaker in Type III Nrgl ${ }^{+/-}$mice. (a) Grand average LFP bursts ( \pm SEM) from the $v H P C$ (top panel) and nACC (bottom panel) from WT (black lines) and Type III Nrgl I ${ }^{+-}$(gray lines) mice. (b) Mean \pm SEM amplitude of LFP bursts recorded from the VHPC (top panel) and nACC (bottom panel) in WT (white bars) and Type III Nrgl ${ }^{+}$- (gray bars) mice at both the trough and peak of the burst. (c) Example cross-correlations of low-frequency $\left(0-50 \mathrm{~Hz}\right.$ ) amplitude between the nACC and vHPC in WT (top) and Type III Nrg I ${ }^{+/-}$(bottom). Peak lags (dotted vertical lines) occur at negative values, indicating that changes in vHPC power precede changes in nACC power. (d) Mean \pm SEM lag between vHPC and nACC, calculated from the peak of the cross-correlations of low-frequency power in the nACC and vHPC. Difference is nonsignificant (see text). $* p<0.05$, WT vs Nrgl ${ }^{+/-}$.

shown to influence neuronal firing in a variety of hippocampal downstream target regions in awake animals (Seidenbecher et al, 2003; Jones and Wilson, 2005; DeCoteau et al, 2007), although the relevance of the LFP bursts reported here to awake $\theta$ oscillations is unclear. In both genotypes, nACC neuron firing was significantly modulated by the $\theta$-frequency component of the vHPC LFP bursts. Figure 6a shows the effect of this modulation: spikes from individual nACC cells that occur during LFP bursts are more likely to fire at specific phases of the $\theta$-frequency components of the vHPC bursts, that is, they tend to be 'phase-locked' to the vHPC LFP. The strength of this phase-locking can be quantified by computing the Rayleigh's Z-statistic (see Materials and Methods); strong phase-locking results in higher $Z$-values. On average, nACC cells from Type III $\mathrm{Nrg}^{+}{ }^{-}$mice were more weakly phase-locked than those from WT mice (Figure 6b), consistent with the hypothesis of decreased functional connectivity between these two brain regions.

The cross-correlation of LFP amplitude envelopes suggests a predominance of vHPC to nACC directionality; the data are consistent with the notion that nACC activity follows activity in the vHPC. If this directionality were indeed accurate, then nACC cell discharge should be most strongly phase-locked to vHPC activity of the recent past (Jones and Wilson, 2005; Siapas et al, 2005). To examine this, phase-locking strength was computed after shifting the spike trains of nACC units in time, both backwards and forwards, relative to the vHPC LFP (Figure $6 c$ and d). Spike trains were shifted in $2.5 \mathrm{~ms}$ steps over lags ranging from -100 to $+100 \mathrm{~ms}$. In WT animals, 13 of $21 \mathrm{nACC}$ neurons were significantly phase-locked at any lag using conservative criteria $(p<0.05$ after Bonferroni correction for multiple comparisons). As predicted, the majority of cells phase-locked most strongly to vHPC LFP of the past, with a mean time shift at maximal Rayleigh's $Z$ of $-17 \mathrm{~ms}$ (Figure 6c). In the Type III Nrg1 ${ }^{+/-}$mice, consistent with the weaker phase-locking noted above, none of the neurons were significantly phase-locked after Bonferroni correction. Nonetheless, nACC units from Type III $\mathrm{Nrg}^{+/-}$mice phase-locked more strongly to the vHPC LFP of the past (mean time shift $-20 \mathrm{~ms}$, Figure $6 \mathrm{~d}$ ). Therefore, in animals of either genotype, nACC unit activity follows vHPC LFP activity. These results confirm the directionality within the vHPC/nACC circuit as suggested by the LFP results. Taken together with the evidence of decreased strength of phaselocking in the Type III $\mathrm{Nrg} 1^{+/-}$mice, these results are consistent with a deficit in functional connectivity between the vHPC and nACC in vivo.

\section{DISCUSSION}

Pursuant to the hypothesized role of circuits involving the nACC in complex behaviors disrupted in schizophrenia, we examined activity in the $\mathrm{ACC}$ and $\mathrm{VHPC}$ regions in vivo in mice carrying a heterozygous mutation in a gene that has been associated with increased susceptibility to schizophrenia, Nrg1. Both WT and Type III Nrg1 ${ }^{+/-}$mice display bursts of synchronized activity in these regions, and the temporal pattern of this synchronized activity suggests that nACC activity followed that in the vHPC. In the Type III $\mathrm{Nrg} 1^{+/-}$mice, the temporal structure of the nACC discharge was significantly different from that recorded in WT animals, and the strength of the modulation of nACC spiking by vHPC activity was significantly reduced. These findings demonstrate a role for Type-III Nrg 1 in regulating function within the vHPC/nACC circuit, and raise the possibility that decreased functional connectivity between the vHPC and nACC may underlie the sensory-motor gating deficits seen in Type III Nrg1 ${ }^{+/-}$mice (Chen et al, 2008).

Although the precise nature of the spontaneous bursts of activity may be unclear, several lines of evidence suggest 


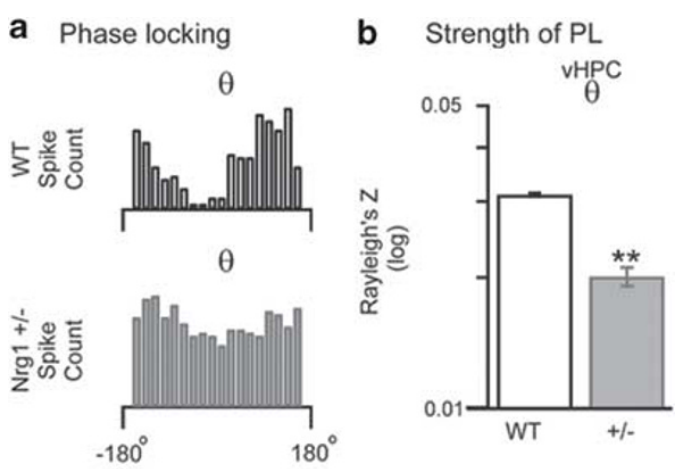

c

WT timeshift analysis
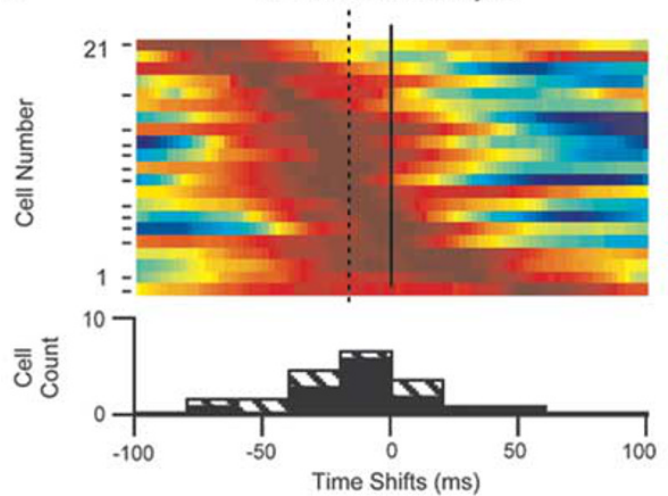

d

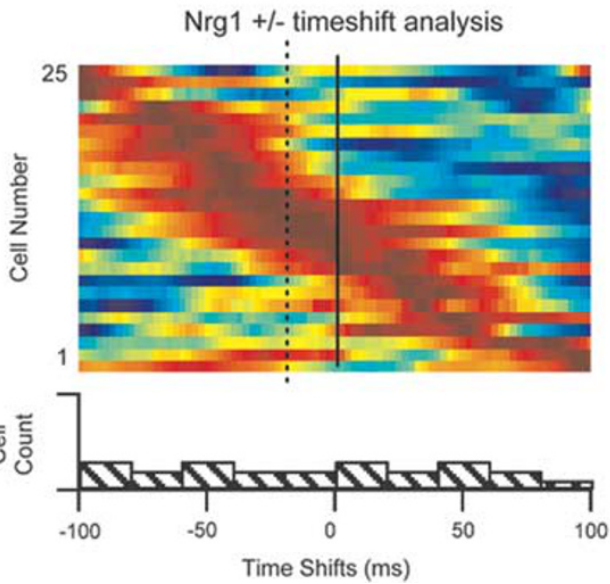

Figure 6 nACC unit response to $\mathrm{VHPC}$ input is weaker in $\mathrm{Nrg} \mathrm{I}^{+/-}$ mice. (a) Spiking of representative single units from WT (top) and Type III $\mathrm{Nrgl}^{+l}$ (bottom) neurons as a function of the phase of the $\theta$-frequency component of the LFP bursts recorded in VHPC. (b) Strength of phaselocking (PL) in WT (open bar) and $\mathrm{Nrgl}^{+/-}$(gray bar) nACC units. Mean \pm SEM of the Rayleigh's Z-statistic from of 21 and 25 units in WT and Type III Nrg| ${ }^{+/}$mice, respectively. (c and d) Normalized phase-locking strength of each recorded nACC neuron as a function of lag. Each row represents an individual neuron ordered by the lag at which phase-locking is maximal. Note that nACC units are most strongly modulated by the vHPC $\theta$ phase of the recent past in both WT (c) and Type III Nrgl ${ }^{+-}$(d) mice. Horizontal lines on the left axis indicate units that demonstrate stronger phase-locking than expected by chance $(p<0.05$ after Bonferroni correction). Note that $13 / 2 \mid \mathrm{WT}$ and 0/2| Type III Nrg| ${ }^{+/-}$units are significantly phase-locked by this test. Bottom: Distribution of lags at which each cell was maximally phase-locked. Filled bars represent units with Bonferroni-corrected significant phase-locking. Mean lags at maximal phaselocking are -17.3 and $-19.1 \mathrm{~ms}$ for WT and Type ||| Nrg| ${ }^{+/}$units, respectively. Negative lags indicate phase-locking to the VHPC of the past. *** $p<0.00$ I, Type III Nrg| ${ }^{+/-}$vs WT.

that the disorganization of activity seen in the mutant nACC is the result of diminished functional input from the vHPC to the nACC. First, although the size of the vHPC LFP bursts are the same or larger in Type III $\mathrm{Nrg}^{+/-}$mice when compared with WT, nACC bursts are dramatically smaller, whether measured as absolute amplitude or as the relative increase in low-frequency power. Second, the synchrony between the two areas is decreased in the Type III Nrg1 ${ }^{+/-}$ mice, as measured by the strength of phase-locking of nACC unit spikes to vHPC low-frequency activity. Third, in both genotypes, nACC activity follows that in the vHPC, as demonstrated using both phase-locking and amplitude cross-correlation methods. There are two possible mechanisms by which nACC activity would follow vHPC activity. Either the bursts of activity seen in the nACC are driven directly by input from the vHPC, or both areas are driven by a third area but with different latencies. Given the presence of monosynaptic connections from the vHPC to the nACC (DeFrance et al, 1985), the former explanation is the most parsimonious.

The known biology of Type III Nrg1 is consistent with an important role in the regulation of neural activity in the nACC. Type III Nrg1 is a membrane-bound protein expressed along axons and at presynaptic terminals of specific subsets of neurons (Michailov et al, 2004; Taveggia et al, 2005; Hancock et al, 2008; Zhong et al, 2008). Type III Nrg1 has been shown to induce short- and long-term changes in cellular signaling cascades and activate downstream gene targets acting as both a ligand and a receptor with the ErbB family of tyrosine kinases. Thus, Type III Nrg1-ErbB interactions constitute a prime example of bidirectional signaling in the nervous system (Bao et al, 2003; Hancock et al, 2008; Mei and Xiong, 2008). Type III Nrg1 is expressed in the vHPC, medial prefrontal cortex, and amygdala, all of which send converging inputs onto medium spiny neurons in the nACC (Groenewegen et al, 1999; French and Totterdell, 2002, 2003; Chen et al, 2008) ErbB4 is expressed postsynaptically on nACC medium spiny neurons (Steiner et al, 1999). Nrg1-ErbB4 signaling interactions are important in synapse formation, stabilization, and plasticity (Wolpowitz et al, 2000; Li et al, 2007; Woo et al, 2007), and the expression of Type III Nrg1 in the vHPC is essential to vHPC-nACC synapses in particular. In vitro studies of vHPC slices and nACC neurons, in which vHPC axons from Type III $\mathrm{Nrg}^{-1-}$ (null), ${ }^{+/-}$, or WT mice innervate WT nACC neurons, demonstrate that functional synapses are formed regardless of presynaptic genotype. However, examination of nascent synapses formed by vHPC slices from Type III $\mathrm{Nrg}^{+/-}$mice and postsynaptic WT nACC neurons in vitro reveals differences in the synaptic profile compared with their WT vHPC/ WT nACC counterparts (lower release probability, altered profile of postsynaptic glutamate receptor channels; C Du and LW Role, unpublished data). These data are consistent with the hypothesis that decreased Nrg1 signaling at vHPC-nACC synapses may underlie the deficits in in vivo functional connectivity described here.

We find that vHPC activity is relatively preserved but that the nACC response to this activity is diminished in $\mathrm{Nrg} 1^{+/-}$ mice. The locus of the deficit in the heterozygote animals is unclear. This could be because of (1) a presynaptic deficit, reducing release of neurotransmitter onto nACC neurons, 
(2) a postsynaptic deficit, reducing the response of nACC neurons to vHPC release of neurotransmitter, and/or (3) a nACC local circuit deficit, reducing the network response to vHPC input. Higher-resolution recording and analyses of synaptic function are required to address this important issue. However, we do find substantive deficits in vHPC/ nACC circuits, whether pre- and/or postsynaptic in origin. Indeed, ongoing work using in vitro preparations supports the hypothesis that both pre- and postsynaptic are involved in altered synaptic profile of Type III Nrg1 ${ }^{+/-}$vHPC/WT nACC connections (Zhong et al, 2008; and manuscripts in preparation by C Zhong, D Talmage, L Jiang, M Mertz, and L Role).

Although its behavioral relevance is not addressed directly by the current experiments, disorganized nACC activity may have a role in the sensorimotor gating deficits seen in Type III Nrg1 $1^{+/-}$mice in which pre-pulse inhibition of acoustic startle is strongly disrupted (Chen et al, 2008). Findings suggestive of decreased hippocampal-accumbens connectivity have also been described in a neurodevelopmental model of schizophrenia neuropathology characterized by impaired pre-pulse inhibition (Moore et al, 2006). Indeed, functional amygdala, prefrontal cortical, and vHPC connections into the striatum are implicated in the regulation of striatal gating to auditory stimuli (Rosen et al, 1991; Swerdlow et al, 2000).

Understanding the impact of schizophrenia risk alleles on the function of neural systems is critical if the causal pathophysiology of schizophrenia is to be fully elucidated. Here we describe the impact of a heterozygous deletion of the cysteine-rich transmembrane domain-containing TypeIII family of isoforms of Nrg1 on the function of the vHPCnACC circuit. We found a disorganization of network activity in the nACC in mice carrying this deletion, and relate this disorganization to decreased functional connectivity between the vHPC and the nACC. The disruption in this circuit may underlie the behavioral deficits in sensorimotor gating, providing an important mechanistic inroad into future investigations into pathophysiological mechanisms known to operate in schizophrenia patients.

\section{ACKNOWLEDGEMENTS}

We thank Mihir Topiwala for technical assistance. MWN is a 2009 NARSAD Sidney R Baer, Jr Foundation Young Investigator. This work was supported by $\mathrm{NIH}$ K08 MH098623 and R01 MH081968 to JAG, a McKnight Brain Disorders and NARSAD Distinguished Investigator Awards to LWR, and the Lieber Center for Schizophrenia Research and Treatment.

\section{DISCLOSURE}

The authors declare no conflict of interest.

\section{REFERENCES}

Adhikari A, Sigurdsson T, Topiwala MA, Gordon JA (2010). Cross-correlation of instantaneous amplitudes of field potential oscillations: a straightforward method to estimate the direction- ality and lag between brain areas. J Neurosci Methods 191: 191-200.

Bao J, Wolpowitz D, Role LW, Talmage DA (2003). Back signaling by the Nrg-1 intracellular domain. J Cell Biol 161: 1133-1141.

Barch DM (2005). The cognitive neuroscience of schizophrenia. Annu Rev Clin Psychol 1: 321-353.

Bjarnadottir M, Misner DL, Haverfield-Gross S, Bruun S, Helgason VG, Stefansson $\mathrm{H}$ et al. (2007). Neuregulin1 (NRG1) signaling through Fyn modulates NMDA receptor phosphorylation: differential synaptic function in NRG1 \pm knock-outs compared with wild-type mice. J Neurosci 27: 4519-4529.

Braff DL, Geyer MA (1990). Sensorimotor gating and schizophrenia. Human and animal model studies. Arch Gen Psychiatry 47: 181-188.

Chen Y, Hancock ML, Role LW, Talmage DA (2010). Intramembranous valine linked to schizophrenia is required for neuregulin 1 regulation of the morphological development of cortical neurons. J Neurosci 30: 9199-9208.

Chen YJ, Johnson MA, Lieberman MD, Goodchild RE, Schobel S, Lewandowski $\mathrm{N}$ et al. (2008). Type III neuregulin-1 is required for normal sensorimotor gating, memory-related behaviors, and corticostriatal circuit components. J Neurosci 28: 6872-6883.

Cooper DC, White FJ (2000). L-type calcium channels modulate glutamate-driven bursting activity in the nucleus accumbens in vivo. Brain Res 880: 212-218.

DeCoteau WE, Thorn C, Gibson DJ, Courtemanche R, Mitra P, Kubota Y et al. (2007). Oscillations of local field potentials in the rat dorsal striatum during spontaneous and instructed behaviors. J Neurophysiol 97: 3800-3805.

DeFrance JF, Marchand JF, Sikes RW, Chronister RB, Hubbard JI (1985). Characterization of fimbria input to nucleus accumbens. J Neurophysiol 54: 1553-1567.

Freedman R, Olincy A, Ross RG, Waldo MC, Stevens KE, Adler LE et al. (2003). The genetics of sensory gating deficits in schizophrenia. Curr Psychiatry Rep 5: 155-161.

French SJ, Totterdell S (2002). Hippocampal and prefrontal cortical inputs monosynaptically converge with individual projection neurons of the nucleus accumbens. J Comp Neurol 446: 151-165.

French SJ, Totterdell S (2003). Individual nucleus accumbensprojection neurons receive both basolateral amygdala and ventral subicular afferents in rats. Neuroscience 119: 19-31.

Goto Y, O'Donnell P (2001a). Network synchrony in the nucleus accumbens in vivo. J Neurosci 21: 4498-4504.

Goto Y, O’Donnell P (2001b). Synchronous activity in the hippocampus and nucleus accumbens in vivo. J Neurosci 21: RC131.

Grace AA (2000). Gating of information flow within the limbic system and the pathophysiology of schizophrenia. Brain Res Brain Res Rev 31: 330-341.

Groenewegen HJ, Trimble M (2007). The ventral striatum as an interface between the limbic and motor systems. CNS Spectr 12: 887-892.

Groenewegen HJ, Vermeulen-Van der Zee E, te Kortschot A, Witter MP (1987). Organization of the projections from the subiculum to the ventral striatum in the rat. A study using anterograde transport of Phaseolus vulgaris leucoagglutinin. Neuroscience 23: 103-120.

Groenewegen HJ, Wright CI, Beijer AV, Voorn P (1999). Convergence and segregation of ventral striatal inputs and outputs. Ann N Y Acad Sci 877: 49-63.

Hancock ML, Canetta SE, Role LW, Talmage DA (2008). Presynaptic type III neuregulin1-ErbB signaling targets \{alphal\}7 nictotinic acetylcholine receptors to axons. J Cell Biol 181: 511-521.

Harrison PJ, Weinberger DR (2005). Schizophrenia genes, gene expression, and neuropathology: on the matter of their convergence. Mol Psychiatry 10: 40-68, image 45. 
Harrison PJ, Law AJ (2006). Neuregulin 1 and schizophrenia: genetics, gene expression, and neurobiology. Biol Psychiatry 60: 132-140.

Jones MW, Wilson MA (2005). Theta rhythms coordinate hippocampal-prefrontal interactions in a spatial memory task. PLoS Biol 3: e402.

Krivosheya D, Tapia L, Levinson JN, Huang K, Kang Y, Hines R et al. (2008). ErbB4-neuregulin signaling modulates synapse development and dendritic arborization through distinct mechanisms. J Biol Chem 283: 32944-32956.

Li B, Woo RS, Mei L, Malinow R (2007). The neuregulin-1 receptor erbB4 controls glutamatergic synapse maturation and plasticity. Neuron 54: 583-597.

Li D, Collier DA, He L (2006). Meta-analysis shows strong positive association of the neuregulin 1 (NRG1) gene with schizophrenia. Hum Mol Genet 15: 1995-2002.

Lopez-Bendito G, Cautinat A, Sanchez JA, Bielle F, Flames N, Garratt AN et al. (2006). Tangential neuronal migration controls axon guidance: a role for neuregulin-1 in thalamocortical axon navigation. Cell 125: 127-142.

Mei L, Xiong WC (2008). Neuregulin 1 in neural development, synaptic plasticity and schizophrenia. Nat Rev Neurosci 9: 437-452.

Michailov GV, Sereda MW, Brinkmann BG, Fischer TM, Haug B, Birchmeier C et al. (2004). Axonal neuregulin-1 regulates myelin sheath thickness. Science 304: 700-703.

Moore H, Jentsch JD, Ghajarnia M, Geyer MA, Grace AA (2006). A neurobehavioral systems analysis of adult rats exposed to methylazoxymethanol acetate on E17: implications for the neuropathology of schizophrenia. Biol Psychiatry 60: 253-264.

Mulder AB, Hodenpijl MG, Lopes da Silva FH (1998). Electrophysiology of the hippocampal and amygdaloid projections to the nucleus accumbens of the rat: convergence, segregation, and interaction of inputs. J Neurosci 18: 5095-5102.

Munafo MR, Thiselton DL, Clark TG, Flint J (2006). Association of the NRG1 gene and schizophrenia: a meta-analysis. Mol Psychiatry 11: 539-546.

Nicodemus KK, Law AJ, Luna A, Vakkalanka R, Straub RE, Kleinman JE et al. (2009). A $5^{\prime}$ promoter region SNP in NRG1 is associated with schizophrenia risk and type III isoform expression. Mol Psychiatry 14: 741-743.

O’Donnell P, Grace AA (1998). Dysfunctions in multiple interrelated systems as the neurobiological bases of schizophrenic symptom clusters. Schizophr Bull 24: 267-283.

O’Donnell P, Greene J, Pabello N, Lewis BL, Grace AA (1999). Modulation of cell firing in the nucleus accumbens. Ann $N Y$ Acad Sci 877: 157-175.

Pennartz CM, Kitai ST (1991). Hippocampal inputs to identified neurons in an in vitro slice preparation of the rat nucleus accumbens: evidence for feed-forward inhibition. J Neurosci 11: 2838-2847.

Rosen JB, Hitchcock JM, Sananes CB, Miserendino MJ, Davis M (1991). A direct projection from the central nucleus of the amygdala to the acoustic startle pathway: anterograde and retrograde tracing studies. Behav Neurosci 105: 817-825.

Seidenbecher T, Laxmi TR, Stork O, Pape HC (2003). Amygdalar and hippocampal theta rhythm synchronization during fear memory retrieval. Science 301: 846-850.

Sesack SR, Grace AA (2010). Cortico-basal ganglia reward network: microcircuitry. Neuropsychopharmacology 35: 27-47.

Siapas AG, Lubenov EV, Wilson MA (2005). Prefrontal phase locking to hippocampal theta oscillations. Neuron 46: 141-151.

Simpson EH, Kellendonk C, Kandel E (2010). A possible role for the striatum in the pathogenesis of the cognitive symptoms of schizophrenia. Neuron 65: 585-596.

Stefansson H, Sigurdsson E, Steinthorsdottir V, Bjornsdottir S, Sigmundsson T, Ghosh S et al. (2002). Neuregulin 1 and susceptibility to schizophrenia. Am J Hum Genet 71: 877-892.

Steiner H, Blum M, Kitai ST, Fedi P (1999). Differential expression of ErbB3 and ErbB4 neuregulin receptors in dopamine neurons and forebrain areas of the adult rat. Exp Neurol 159: 494-503.

Swerdlow NR, Braff DL, Geyer MA (2000). Animal models of deficient sensorimotor gating: what we know, what we think we know, and what we hope to know soon. Behav Pharmacol 11: 185-204.

Taveggia C, Zanazzi G, Petrylak A, Yano H, Rosenbluth J, Einheber $S$ et al. (2005). Neuregulin-1 type III determines the ensheathment fate of axons. Neuron 47: 681-694.

Thaker GK (2000). Defining the schizophrenia phenotype. Curr Psychiatry Rep 2: 398-403.

Wolpowitz D, Mason TB, Dietrich P, Mendelsohn M, Talmage DA, Role LW (2000). Cysteine-rich domain isoforms of the neuregulin-1 gene are required for maintenance of peripheral synapses. Neuron 25: 79-91.

Woo RS, Li XM, Tao Y, Carpenter-Hyland E, Huang YZ, Weber J et al. (2007). Neuregulin-1 enhances depolarization-induced GABA release. Neuron 54: 599-610.

Wright CI, Groenewegen HJ (1995). Patterns of convergence and segregation in the medial nucleus accumbens of the rat: relationships of prefrontal cortical, midline thalamic, and basal amygdaloid afferents. J Comp Neurol 361: 383-403.

Zhong C, Du C, Hancock M, Mertz M, Talmage DA, Role LW (2008). Presynaptic type III neuregulin 1 is required for sustained enhancement of hippocampal transmission by nicotine and for axonal targeting of alpha7 nicotinic acetylcholine receptors. J Neurosci 28: 9111-9116.

Supplementary Information accompanies the paper on the Neuropsychopharmacology website (http://www.nature.com/npp) 